\title{
Stellar p-modes oscillation measurements in solar-like star signals: $\alpha$ Centauri A as an example
}

\author{
C. Régulo ${ }^{1,2}$ and T. Roca Cortés ${ }^{1,2}$ \\ 1 Instituto de Astrofísica de Canarias, 38205 La Laguna, Tenerife, Spain \\ 2 Dpto. de Astrofísica, Universidad de La Laguna, 38206 La Laguna, Tenerife, Spain \\ e-mail: [crr;trc]@iac.es
}

Received 6 July 2006 / Accepted 28 January 2007

ABSTRACT

\begin{abstract}
Context. This paper deals with the analysis of observational data series of solar-like stars. In dealing with observations of solar-like stellar oscillations from ground observatories, to achieve the necessary level of signal to noise to measure individual acoustic modes in their power spectra it is necessary to have access to large telescopes and preferably more than one used simultaneously at appropriate longitudes. It is very difficult to obtain observing time to cover more than a few day series of data, which usually implies difficulties in obtaining a precise determination of the acoustic frequencies.

Aims. To find the acoustic frequencies of solar-like stars from short duration data series.

Methods. The analysis technique is based on an earlier work by the authors that allows the recovery of the acoustic power spectrum of solar-like stars even in cases with poor $\mathrm{S} / \mathrm{N}$ ratios. The method is applied to solar data to show how it works and also it is used to re-analyse the recently published ultra-high-precision velocity measurements in $\alpha$ Cen A.

Results. The method is shown to work well with solar observations and allows us to obtain the acoustic frequencies of solar-like stars from short-duration data series with a precision comparable with that obtained from standard methods in longer data series.

Conclusions. This method can be used with currently observed data series to provide useful information on the structure of stars.
\end{abstract}

Key words. stars: oscillations - stars: individual: $\alpha$ Cen A

\section{Introduction}

The small amplitude of the acoustic modes in solar-like stars is the reason why a reliable determination of p-mode frequencies in their power spectra is a very difficult task. From ground-based observatories, the use of reasonably large telescopes is necessary for both photometric or spectrometric techniques (Harvey 1988); moreover, to avoid the noise introduced by the window function when the observations are made from only one site, it is convenient to have data from at least two simultaneously well separate telescopes. However, another requirement is to have long data sets to separate the modes properly. To obtain these kinds of telescopes for long periods of time (more than a week) is usually as difficult as the determination of the modes.

A straightforward method to determine the p-mode frequencies of solar-like stars using short-duration series of data with a good precision comparable with the obtained from longer data sets is the proposed goal of this paper. The method used is the one developed in Régulo \& Roca Cortés (2002), which is of special interest when the signal is buried in noise (Régulo \& Roca Cortés 2005), but as it allows the recovery of the original power spectrum from a knowledge of the frequency spacing present in the data, it is also very useful for unambiguous identification of the frequency modes and allows us to achieve a measured set of frequencies with the highest possible internal consistency; namely, being globally as close as possible to those expected from the star.

To show how the method works and its improvements in the determination of $\mathrm{p}$-mode oscillations in solar-like star signals, solar data taken with GOLF aboard SoHO are used. Next, the method is applied to the recently published ultra-high-precision velocity measurements in $\alpha$ Cen A (Butler et al. 2004). $\alpha$ Cen A, a G2 star similar to the Sun, is the second closest star to us and the brightest component of the triple star system $\alpha$ Cen which is only 4.3 light-years away. For these reasons it has been an object of special interest for astronomy for centuries. From the point of view of asteroseismology, $\alpha$ Cen $\mathrm{A}$ is a very interesting target too, since it is a solar twin. In fact, seismological approaches to obtaining the acoustic oscillation power spectrum of this star cover two decades since the first by Gelly et al. (1986). However, before the observations by Bouchy \& Carrier (2001), no unambiguous determination of its oscillations were achieved. After that, two impressive sets of data have been collected (Bouchy \& Carrier 2002; Butler et al. 2004).

The set of data re-analysed in this paper to obtain the oscillation frequencies of this star is one of the best sets of asteroseismic data available for $\alpha$ Cen A. Its quality is impressive, with the discrete peaks clearly visible in the power spectrum of the data well above the noise level. However, as the time span of the data is only 4.5 days, the theoretical resolution is $2.5 \mu \mathrm{Hz}$ and a precise identification of the modes and determination of their frequencies is difficult. For this reason this is a perfect example of a data set for checking whether the method proposed in this paper works, allowing an unambiguous identification of the peaks and a good determination of the frequencies, which is crucial when they are used to model the structure of the star.

Section 2 is dedicated to data analysis: the solar data in Sect. 2.1 and the stellar data in Sect. 2.2. In Sect. 3, a discussion of the results is presented. 


\section{Data analysis}

In this section, two sets of data are analysed. The first consists of 100 days of solar full disk Doppler velocity observations obtained with GOLF instrument on board SoHO satellite (Gabriel et al. 1995). The data set covers the period from April 1996 to July 1996, in the minimum of the solar cycle, and has a cadence of $60 \mathrm{~s}$ with a duty cycle higher than $99 \%$. The analysis of these data is also used to recall the applied methodology. The second set is the ultra-high precision velocity measurements of oscillations in $\alpha$ Cen A obtained by Butler et al. (2004), kindly provided by the team. The observations were made in May 2001 at the European Southern Observatory in Chile using the UVES (UV-Visual Echelle Spectrograph) at $8.2 \mathrm{~m}$ Unit Telescope 2 of the Very Large Telescope and simultaneously at the Siding Spring Observatory in Australia with the UCLES (University College London Echelle Spectrograph) at the $3.9 \mathrm{~m}$ Anglo Australian Telescope (AAT). The spectra were collected at a cadence of around 26 and $20 \mathrm{~s}$ respectively during 4.47 days.

When a set of oscillation frequencies of a star are obtained from a given period of observational data that is short compared to the lifetime of the modes, they may differ from the "real frequencies" of the star due to the stochastic nature of the excitation of the oscillations and their damping time. Here, we call "real frequencies" those that can be obtained in the limit of a very long set of data. In general, we do not yet know the "real frequencies" of a star except for the Sun.

\subsection{Solar data}

The 100 days of solar data analysed in this section are the first of a series of 805 days obtained during the minimum of the solar activity cycle by the GOLF instrument and analysed in several papers, the latest being Gelly et al. (2002), to obtain the p-mode frequencies, among other parameters. Such a p-mode frequency set obtained by Gelly et al. (2002) from more than 2 years of continuous data (the longer possible series before the frequencies start to change due to the increase of the solar activity), measured from a satellite and with a duty cycle of $99.35 \%$, will be taken as the "real frequencies" of the Sun when it is in its lower level of activity.

The first 100 days of this series of 805 days have been used to analyse the effect, in the determination of p-modes frequencies, of having a short duration set of data instead of a long one. First, the 100 days of data have been split in 22 independent sets of 4.5 days each (approximately the length we have for the $\alpha$ Cen A data set). Second, the frequencies of 33 p-modes with $\ell=0,1$ and 2 and $n$ between 15 and 26 were analysed in each of these sets of 4.5 days of data.

Although the data sampling is constant while the data from $\alpha$ Cen A are not, to follow exactly the same procedure that is going to be used to analyse the stellar data, the power spectrum of the 4.5-day data series of solar data were obtained using an iterative sine wave-fitting procedure (SWF) instead of a fast Fourier transform (FFT). The method consists of fitting a simple sine wave function $f(t)=\alpha \sin (2 \pi v t)+\beta \cos (2 \pi v t)$ at any given $v$ to the data. From $\alpha$ and $\beta$, the amplitude and phase of the fitted function are obtained. The scanned frequency range covers from $2000 \mu \mathrm{Hz}$ to $4000 \mu \mathrm{Hz}$ ( $n$ between 15 and 26) in steps of $0.1 \mu \mathrm{Hz}$, well below the frequency resolution provided by the data length (4.5 days). From these 22 power spectra, each p-mode in each power spectrum was fitted to a Lorentzian symmetric profile using a standard nonlinear least-squares method. The whole spectrum was fitted at once in two blocks, the
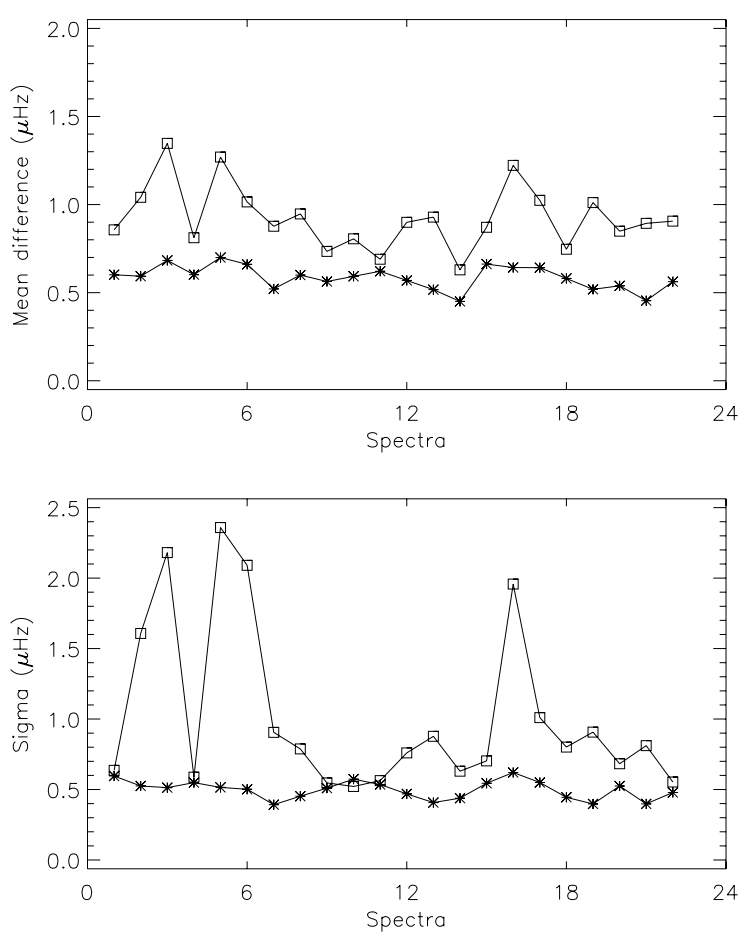

Fig. 1. Average value (top) and standard deviation (bottom) of the absolute value of the differences between the 33 obtained solar p-modes frequencies and the "real frequencies" of the solar p-modes for each of the 22 analysed spectra. Squares represent the results obtained from the direct fit to the spectra and asterisks those obtained from the fit to the recovered spectra using the methodology proposed here.

first part between 2200 and $2800 \mu \mathrm{Hz}$ and the second one between 2800 and $3800 \mu \mathrm{Hz}$. This procedure provides us with 22 independently obtained frequency sets of $\mathrm{p}$-mode frequencies of the Sun. For each of the 22 sets of data, 33 frequencies are determined $(l=0,1$, and 2$)$ as said before. The absolute value of the difference between each of these 33 frequencies and the 33 "real frequencies" (from Gelly et al. 2002) are calculated; the mean value and the standard deviation of these 33 values are taken as a mesaure of the "precision" of the global frequency set obtained and are shown as squares in Fig. 1 for the 22 analysed spectra.

Further, other sets of frequencies were obtained again for the same power spectra, but using the method proposed in Régulo $\&$ Roca Cortés (2002) for the detection of p-mode oscillations in solar-like stellar signals. The method used not only allows the detection of the modes but when detected, it is able to recover the original power spectrum with the only hypothesis that the modes' frequency set is uniformly spaced. The method is based on this regular pattern shown by the power spectrum of solar-like star acoustic oscillations, according to the asymptotic theory for such oscillations in these type of stars (e.g. see Tassoul 1980), as amply verified by observations. In brief, the method works as follows: the starting point for the analysis is the power spectrum of the signal; then, the frequency interval in the power spectrum where the signal is expected to lie is selected and its power spectrum is calculated. This second power spectrum, where there is a set of peaks equally spaced, with the first one being at zero frequency, is where the frequency spacing $\Delta v\left(\left\langle v_{n, l}-v_{n-1, l}\right\rangle\right)$ is searched for and found. With this obtained spacing, it is possible to go back (inverting the FFT) to find the recovered spectrum of the signal. To do that, in the complex Fourier transform of the power spectrum of the signal, all equally spaced peaks are selected and the inverse Fourier transform applied. The 
result is a recovered power spectrum of the stellar oscillation observations with better $\mathrm{S} / \mathrm{N}$ and cleaner peaks but with different amplitudes or energies. It is important to note, as was shown in Régulo \& Roca Cortés (2002), that if the selected bins used to recover the power spectrum are not generated by a signal present in the data, their phases do not have the correct relationship to reconstruct the signal and a noise spectrum is recovered.

Moreover, the method can distinguish between different spacings at different intervals if they exist, particularly if the spacing between peaks varies slightly with frequency, as is the case for the Sun, the method can recover such variation by analysing different intervals in the power spectrum. In our present case, we have selected two frequency intervals to analyse to mimic the search carried out for $\alpha$ Cen A. The interval are from $n=15$ to $n=18$ and from $n=19$ to $n=26$. The spacings found were $134.1 \mu \mathrm{Hz}$ and $135.2 \mu \mathrm{Hz}$ respectively. With these two spacings, the power spectrum was recovered for each of the 22 independent data series and the frequencies were obtained by fitting each p-mode to a Lorentzian symmetric profile as explained before for the direct fit. The average value and the standard deviation of the absolute value of the differences between the 33 obtained frequencies from each recovered spectra and the "real frequencies" of the Sun are plotted in Fig. 1 (asterisks) for each of the 22 analysed spectra.

As is clear from this exercise, both the average and the standard deviation are smaller for the frequencies obtained from the recovered spectra, showing that these sets of frequencies are closer to the frequencies obtained in the limit of a long set of data; thus being consistently more suitable for use as the "real frequencies" than those obtained directly from any of the single spectra of the short-length series. It is important to remark that not only is the mean values of the difference smaller but the standard deviation is smaller too. The mean value of the average of these absolute differences is $0.93 \mu \mathrm{Hz}$ in the case of direct detection and $0.58 \mu \mathrm{Hz}$ when our method is applied, $1.02 \mu \mathrm{Hz}$ and $0.50 \mu \mathrm{Hz}$ being the mean values of the obtained sigmas.

The exercise was repeated using "observed data" twice as long as those used previously. In this case, the first 200 days of the series of 805 days were split into series of 9 independent days as "observed data length" yielding results that are closer to each other and closer to those taken as "real frequencies". Now the mean value of the absolute average differences to the "real frequencies" is $0.74 \mu \mathrm{Hz}$ for the case of a direct fit and $0.56 \mu \mathrm{Hz}$ when our method is applied with mean values of $0.81 \mu \mathrm{Hz}$ and $0.48 \mu \mathrm{Hz}$ for the sigmas. These numbers suggest that, as expected with long enough data sets, both ways of obtaining the frequencies will converge to those defined as "real frequencies".

\section{2. $\alpha$ Cen A data}

The data used in this section are the ultra-high precision velocity measurements of oscillations in $\alpha$ Cen A obtained by Butler et al. (2004). The spectra were collected at a cadence of around 26 and $20 \mathrm{~s}$ respectively during 4.47 days, therefore yielding a frequency resolution of $2.59 \mu \mathrm{Hz}$.

In this paper, the velocity data are analysed with optimum weights adjusted by Butler et al. (2004) in order to optimize the observing window function effect, which finally yield sidelobes of the modes in the power spectrum of the data of only $3.6 \%$ in power of the main peak. Since the final time series is not equally sampled in time, the power spectrum of the combined data were obtained using an iterative sine wave fitting procedure (SWF) instead of an FFT. The scanned frequency range covers from $1500 \mu \mathrm{Hz}$ to $3500 \mu \mathrm{Hz}$ in steps of $0.1 \mu \mathrm{Hz}$. The obtained

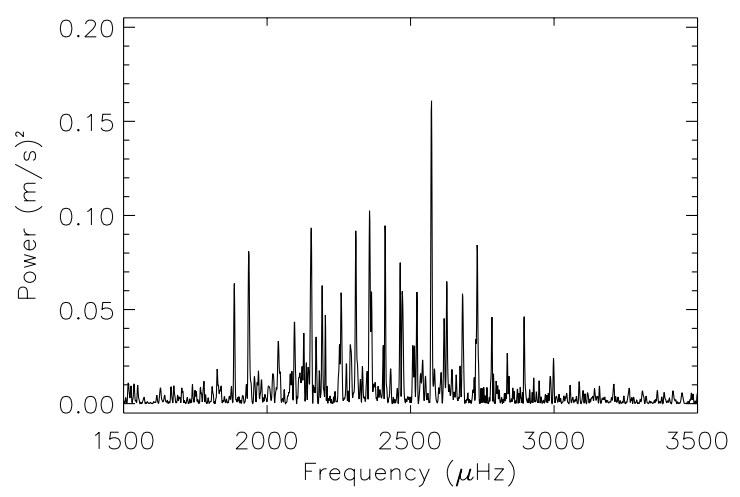

Fig. 2. Power spectrum of $\alpha$ Cen A (4.5 days of combined data from Chile and Australia).

spectrum is shown in Fig. 2. This power spectrum is directly comparable with Fig. 1 in Bedding et al. (2004). Although the quality of the obtained power spectrum is impressive and the p-mode oscillations are clearly visible above the noise, the poor frequency resolution of the data set prevents a precise determination of the frequencies, it being difficult even to resolve the $\ell=$ 0,2 doublets in some of these $\mathrm{p}$-mode couples.

The obtained spectrum was analysed with the same method and the same procedure described in the previous subsection for the solar frequencies.

In this particular case, the frequency interval selected was from 1750 to $3000 \mu \mathrm{Hz}$, where the signal is clearly visible. From the power spectrum of this selected range, a spacing of $106.2 \mu \mathrm{Hz}$ was obtained. However, to see if a possible change in the spacing exists in the data, smaller ranges were analysed from the power spectrum of the signal to find the spacing again. The result was that between 1750 and $2000 \mu \mathrm{Hz}$, the spacing found was $105.5 \mu \mathrm{Hz}$, which agrees with that obtained by Bouchy \& Carrier (2002), whereas from 2000 to $3000 \mu \mathrm{Hz}$ it was $106.2 \mu \mathrm{Hz}$, which agrees with that given by Bedding et al. (2004) between 2020 and $2970 \mu \mathrm{Hz}$. Due to the limited frequency interval where p-modes with enough $\mathrm{S} / \mathrm{N}$ are present, only these two values for the spacing could be found. Notice that both spacings are within the frequency resolution of the data set. With these two spacings, the power spectrum is recovered, as explained in the previous subsection. The spacing of $105.5 \mu \mathrm{Hz}$ was used between 1750 and $2100 \mu \mathrm{Hz}$ and $106.2 \mu \mathrm{Hz}$ from 2000 to $3000 \mu \mathrm{Hz}$. These obtained spacings agree with most of the proposed models for $\alpha$ Cen A, where $\Delta v$ is almost constant in the range from 2000 to $3000 \mu \mathrm{Hz}$ with an oscillatory behaviour below $2000 \mu \mathrm{Hz}$ related with the helium ionization zone (Yong-Cheol 1999; Provost et al. 2001; Thoul et al. 2003), which yields a decrease of $\Delta v$ in the range between 1750 and $2000 \mu \mathrm{Hz}$. This is what it is found in our analysis of the data, justifing the use of two constant $\Delta v$ 's to recover the power spectrum of the signal in the ranges where we have used them.

The recovered spectrum from 2000 to $3000 \mu \mathrm{Hz}$ is shown in Fig. 3, where the $\ell=0,2$ doublets are well visible and resolved; the $\ell=1 \mathrm{p}$-modes stand up very clearly too, whereas the $\ell=3 \mathrm{p}$-modes are not recovered. As has been said previously (Sect. 2.1), the method can recover the signal in the power spectrum when the frequency bins selected keep their correct phases; if the interference between the signal and the noise is such that the phases are too contaminated, the original signal is not recovered, which is the case for $\ell=3$ modes. Moreover, these $\ell=3$ p-modes are very close to $\ell=1$ ( 3 to 4 bins), which 


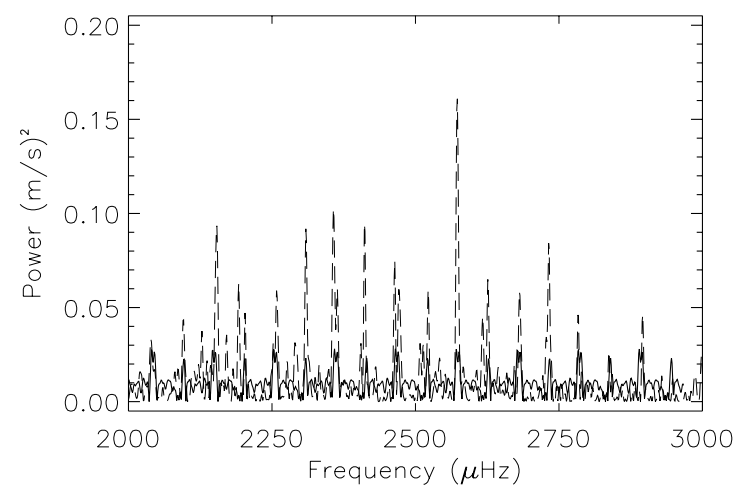

Fig. 3. Recovered power spectrum of $\alpha$ Cen A using the method developed in Régulo \& Roca Cortés (2002). The recovered power spectrum (thick line) is plotted over the original one (dashed line) for comparison.

have a power at least 9 times higher (Christensen-Dalsgaard \& Gough 1982); this will make the case still worse. To obtain the frequencies of the recovered p-modes, each p-mode in this power spectrum was fitted to a Lorentzian symmetric profile as for the solar data. The whole spectrum is fitted at once in two blocks: the first part between 1750 and $2100 \mu \mathrm{Hz}$ and the second one between 2000 and $3000 \mu \mathrm{Hz}$. As for $n=18 \ell=0, n=18 \ell=1$ and $n=17 \ell=2 \mathrm{p}$-modes, two values of the frequencies have been obtained because they are the modes where the spacing changes, the mean value is accepted as the best approximation. The results of these fits are in Table 1 where the differences with the results obtained by Bedding et al. (2004) and Bouchy \& Carrier (2002), the current best $\alpha$ Cen A frequency sets, are also shown for comparison.

Notice that obviously the frequencies of the well identified peaks follow the first-order asymptotic relation (Tassoul 1980):

$v(n, \ell) \approx \Delta v(n+\ell / 2+\epsilon)$.

It is therefore possible to identify the radial order $n$ of the modes and to calculate the value of $\epsilon$, which is related with the equilibrium structure close to the star's surface. The so-called large frequency difference $(\Delta v)$ is the frequency spacing among peaks of a given $\ell$ value obtained directly with the method applied. Moreover, the small frequency difference between the $\ell=0$ and the closest $\ell=2$ modes $\left(\delta v_{02}=v_{n, \ell}-v_{n-1, \ell+2}\right.$, a second order effect) can also be calculated from the obtained frequencies (see Table 2). Such a small difference is directly related to properties in the core of the star.

In order to test possible misidentifications among the obtained frequencies, their ratio, $v_{n} / v_{n+1}$, for a given $\ell$ value is plotted vs. the large spacing $\Delta v$ in Fig. 4 . As developed in Régulo et al. (2003), when the ratio of consecutive observed frequencies of the same degree is plotted in this type of diagram, they must show a regular pattern where any misidentification in the peaks is clearly reflected in a departure from the expected ratios calculated from close stellar models. In Fig. 4, the flat lines represent the theoretical frequency ratios for the model of $\alpha$ Cen A proposed by Thoul et al. (2003). The particular model used for comparison with the observed frequencies is not critical because, as shown in Régulo et al. (2003), when the frequency ratio of modes with the same degree $\ell$ and consecutive radial orders $n$, obtained from models of solar-like stars with slightly different masses, are plotted vs. the spacing of the modes $\Delta v$, almost no variation appears (thus the horizontal straight lines). This is due to the weak dependence of this ratio on the mass and age of the star, especially for masses close to solar. In this diagram, the position of the ratio of consecutive observed frequencies must show
Table 1. Oscillation frequencies for $\alpha$ Cen A $(\mu \mathrm{Hz}) . \delta v(1)$ are the differencies $(\mu \mathrm{Hz})$ with the frequencies obtained by Bedding et al. (2004) $(v($ Bedding $)-v)$ and $\delta v(2)$ the differencies $(\mu \mathrm{Hz})$ with the results from Bouchy \& Carrier (2002) ( $v$ (Bouchy) $-v)$.

\begin{tabular}{cccc}
\hline \hline$n$ & $l=0$ & $\delta v(1)$ & $\delta v(2)$ \\
\hline 16 & 1836.33 & 2.9 & 5.0 \\
17 & 1941.81 & 1.5 & - \\
18 & 2046.22 & -0.7 & - \\
19 & 2151.30 & 1.6 & 1.6 \\
20 & 2257.51 & 0.6 & 0.9 \\
21 & 2363.68 & 0.3 & 0.5 \\
22 & 2469.86 & 1.6 & 0.1 \\
23 & 2576.03 & - & -2.9 \\
24 & 2682.20 & 0.5 & -2.4 \\
25 & 2788.38 & - & -2.2 \\
26 & 2894.60 & 1.3 & - \\
\hline$n$ & $l=1$ & $\delta v(1)$ & $\delta v(2)$ \\
\hline 15 & 1780.87 & -1.2 & - \\
16 & 1886.43 & -0.5 & 1.0 \\
17 & 1991.87 & 1.9 & 0.2 \\
18 & 2096.95 & -2.3 & -1.3 \\
19 & 2202.58 & 0.6 & 0.2 \\
20 & 2308.74 & 0.4 & 0.4 \\
21 & 2414.96 & -2.5 & -0.7 \\
22 & 2521.20 & 0.9 & -1.9 \\
23 & 2627.39 & -0.3 & -1.8 \\
24 & 2733.52 & -0.3 & -0.3 \\
25 & 2839.65 & 0.5 & -2.0 \\
26 & 2945.84 & -0.1 & - \\
\hline$n$ & $l=2$ & $\delta v(1)$ & $\delta v(2)$ \\
\hline 15 & 1829.96 & -1.4 & 3.1 \\
16 & 1935.46 & 0.2 & -0.6 \\
17 & 2040.71 & -1.8 & 0.8 \\
18 & 2146.61 & -0.3 & -0.6 \\
19 & 2252.83 & 0.6 & -1.4 \\
20 & 2359.03 & -1.7 & -0.6 \\
21 & 2465.21 & -1.8 & -1.1 \\
22 & 2571.38 & 1.3 & -2.9 \\
23 & 2677.52 & -0.7 & - \\
24 & 2783.70 & -0.3 & -0.8 \\
25 & 2889.93 & - & -2.2 \\
\hline & & & \\
& & & \\
15 & \\
17 &
\end{tabular}

Table 2. Parameters calculated from the obtained frequencies.

\begin{tabular}{cccc}
\hline \hline Range in $\mu \mathrm{Hz}$ & $\Delta v(\mu \mathrm{Hz})$ & $\delta v_{02}(\mu \mathrm{Hz})$ & $\epsilon$ \\
\hline $1750-2000$ & 105.5 & 6.40 & 1.38 \\
$2000-3000$ & 106.2 & 4.65 & 1.24 \\
\hline
\end{tabular}

a very regular pattern that should closely follow the straight horizontal lines of the theoretical values predicted by the models.

This type of diagram yields a simple, "almost visual", method to discriminate peaks misidentified as p-modes; in other words, those that must be taken as suspicious when used in any attempt at modelling the star. The frequencies obtained for $\alpha$ Cen $\mathrm{A}$ in this paper whose ratios are shown as asterisks in Fig. 4, present a very regular pattern following the one predicted by the model. Although they do not exactly coincide with the model, what their values, compared to those of the model, are reflecting is possible inaccuracies in the model used. However, the ratios of the frequency sets obtained by Bouchy \& Carrier (2002) and Bedding et al. (2004), shown as diamonds in Fig. 4, seem to have problems with some of the frequencies of the identified peaks; their set of frequencies show an irregular pattern even having, in some cases, two consecutive ratios, one on top of the other. Although the spacing, $\Delta v$, for our frequencies and 

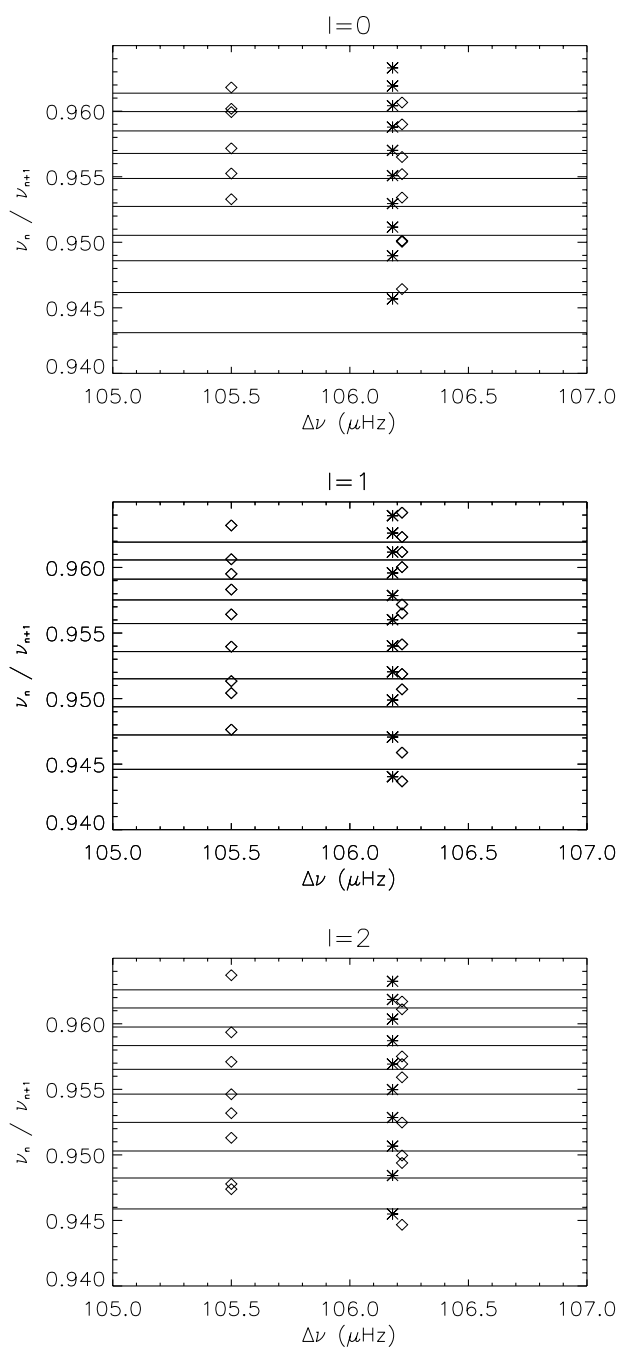

Fig. 4. Frequency ratios of consecutive radial order for $\ell=0$ (top), $\ell=1$ (middle) and $\ell=2$ (bottom) vs. $\Delta v$ for the different sets of $\alpha$ Cen A frequencies discussed in the paper. Diamonds for Bouchy \& Carrier (2002) at $\Delta v=105.5 \mu \mathrm{Hz}$ and Bedding et al. (2004) at $106.2 \mu \mathrm{Hz}$, compared with the results obtained in this paper (asterisks). The continuous lines correspond to the model of $\alpha$ Cen A proposed by Thoul et al. (2003). See text.

for the frequencies of Bedding et al. (2004) are the same, in the plot they have been shifted a little $( \pm 0.02 \mu \mathrm{Hz})$ for clarity; in the same way, and also for clarity in the picture, all our obtained ratios have been plotted at $\Delta v=106.2 \mu \mathrm{Hz}$, to allow the eye to follow a unique line, although the three smallest frequencies of each degree, $\ell$, correspond to a $\Delta v=105.5 \mu \mathrm{Hz}$. Such a diagram shows that the frequencies of the peaks obtained here, as we expect from the way in which they have been obtained, seems to be closer to those expected from the star than previous identifications, which suffer from the scatter due to the noise level and/or to the finite lifetime of the modes and the small number of observed realisations.

\section{Discussion and conclusions}

A method that allows us to overcome the natural scatter of a set of acoustic mode frequencies due to short length of data and/or to the short lifetime of the oscillations has been discussed in this paper. In the proposed method, the frequencies are obtained from the recovered spectrum of the data, a reconstruction based on the previously determined p-mode frequency spacing signature, instead of being obtained directly from the observed spectrum. This method is based on the fact that the acoustic modes of solarlike stars are almost equally spaced in frequency. It is this spacing which is used to reconstruct the power spectrum.

From the method, two different values for the large and the small frequency differences, $\Delta v$ and $\delta v_{02}$, at two different frequency intervals have been obtained for $\alpha$ Cen A (see Table 2), showing that both parameters vary with frequency. The method, applied to these data, provides only two different mean values, which can be compared with those obtained by Bedding et al. (2004). Whereas the large difference, $\Delta v$, agrees exactly with that obtained by Bedding et al. (2004) between 2000 and $3000 \mu \mathrm{Hz}$, the small one, $\delta v_{02}$, can be compared with Eq. (1) in Bedding et al. (2004), where a linear dependence of $\delta v_{02}$ on frequency is used. Notably, our average value in the whole frequency range analysed coincides with theirs to within $3 \%$.

From solar data, we show that the set of frequencies obtained from the recovered spectrum are closer to the "real frequencies" than those obtained directly from the observed spectrum (by "real frequencies" we mean those obtained in the limit of a very long series of data). From the frequencies obtained in $\alpha$ Cen $\mathrm{A}$, we found that our set of 34 oscillation frequencies have differences compared with those obtained by Bedding et al. (2004) that are within plus or minus one independent bin in the power spectrum, but without the scatter problems that the Bedding et al. (2004) frequencies show (see Fig. 4). Such scatter is partially due to the short lifetimes of the modes and the short length of data analysed. While the authors minimized this scatter by appropriate fitting, involving nine free parameters, as the first-order asymptotic theory predicts, the alternative method proposed in this paper simplifies the determination of the frequencies with results globally comparable and even better than those obtained from direct fits to the obtained spectra.

Acknowledgements. The authors wish to thank $\mathrm{H}$. Kjeldsen for providing the $\alpha$ Cen A data used in this paper. We would like also to thank our colleagues: scientists, engineers, and technicians involved with the GOLF project. This work has been partially funded under grants AYA 2001-1571 and ESP 2004-03855-C03-03 of the spanish national research plan.

\section{References}

Bedding, T. R., Kjeldsen, H., Butler, R. P., et al. 2004, ApJ, 614, 380

Bouchy, F., \& Carrier, F. 2001, A\&A, 374, L5

Bouchy, F., \& Carrier, F. 2002, A\&A, 390, 205

Butler, R. P., Bedding, T. R., Kjedsen, H., et al. 2004, ApJ, 600, L75

Christensen-Dalsgaard, J., \& Gough, D. O. 1982, MNRAS, 198, 141

Gabriel, A. H., Grec, G., Charra, J., et al. 1995, Sol. Phys., 162, 61

Gelly, B., Grec, G., \& Fossat, E. 1986, A\&A, 164, 383

Gelly, B., Lazrek, M., Grec, G., et al. 2002, A\&A, 394, 285

Harvey, J. W. 1988, Proc. IAU Symp., 123, 497

Provost, J., Berthomieu, G., Morel, P., Lebreton, Y., \& Thevein, F. 2001, ESA, SP-464, 439

Régulo, C., \& Roca Cortés, T. 2002, A\&A, 396, 745

Régulo, C., \& Roca Cortés, T. 2005, A\&A, 444, L5

Régulo, C., Pérez Hernández, F., \& Roca Cortés, T. 2003, ESA, SP-538, 393

Tassoul, M. 1980, AJS, 43, 469

Thoul, A., Scuflaire, R., Noels, A., et al. 2003, A\&A, 402, 293

Yong-Cheol Kim 1999, J. Korean Astron. Soc., 32, 119 